\title{
ÜBER DIE AUSBREITUNG DER LARVEN DER PHYSOKERMESARTEN, \\ SPEZIELL VON PHYSOKERMES HEMICRYPHUS DALM. (KLEINE FICHTENQUIRLSCHILDLAUS ODER KLEINE LECANIE)
}

\author{
Sur la propagation des larves de Physokermes, \\ en particulier de Physokermes hemicryphus Dalm \\ (Petit Lecanium de l'Epicéa)
}

\section{Hermann PECHHACKER}

Bundes-Lehr-und Versuchsanstalt für Bienenkunde, Wien, Außenstelle Lunz/See.

\section{SUMMARY}

\author{
ON THE PROPAgation OF THE LARVAE OF PHYSOKERMES, \\ especially of Physokermes hemicryphus dalm \\ (SMALL SPRUCE SHIELD LOUSE)
}

The possibilities of propagation of Physokermes hemicryphus Datm. were examined considering the determinative environmental in fluences.

It was shown that,

1. at warm and dry atmospheric conditions only, the primary larvae $\left(L_{1}\right)$ can leave their mother's cover because at moisture and high rel. air humidity, the anal aperture remains closed;

2. besides the already known active propagation a by foot $)$ on a host tree during emerging time (end of July/Sept.), a second migration may occur in spring preceding the last ecdysis;

3. passive propagation by wind, likewise is possible to a high degree. In investigations of the years 1968,1969 and 1970 implemented during one week of fine weather, respectively, we succeeded in finding 14 larvae $L_{1}$ at place $A$ and $18 \mathrm{~L}_{1}$ at place $B, 500 \mathrm{~m}$ distant from the wood. The flattened structure of the primary larvae $L_{1}$ enables them to this propagation. The larvae are hovering to the ground like falling beech leaves at a velocity of $0,54 \mathrm{~m} / \mathrm{sec}$, only. 


\section{ZUSAMMENFASSUNG}

Es wurden die Möglichkeit der Ausbreitung von Physokermes hemicryphus Dalm. und die dabei ausschlaggebenden Umweltein flüsse untersucht. Dabei wurde festgestellt :

1. Die Erstlarven $\left(\mathrm{L}_{1}\right)$ von Physokermes hemicryphus Dalm. haben nur bei warmem und trockenem Wetter die Möglichkeit, die mütterliche Hülle zu verlassen, da (u. a.) der Anal- oder Schlüpfspalt bei Nässe oder hoher rel. Luftfeuchtigkeit geschlossen bleibt.

2. Neben der bereits bekannten aktiven Ausbreitung zu Fuß innerhalb eines schon besetzten Baumes zur Schlupfzeit (Ende Juli bis September) kann auch noch im Frühjahr vor der letzten Häutung eine Wanderung stattfinden.

3. Eine passive Ausbreitung durch den Wind ist in starkem Ausmaß gegeben. So wurden bei Versuchen in den Jahren 1968, 1969 und 1970 im Durchschnitt von jeweils ca. einer Woche Schönwetter pro Fangperiode noch in einer Entfernung von $500 \mathrm{~m}$ vom Wald $14 \mathrm{~L}_{1}$ pro $\mathrm{m}^{2}$ auf Platz A und $18 \mathrm{~L}_{1}$ pro $\mathrm{m}^{2}$ auf Platz B gefangen. Der flache Habitus der Erstlarve ermöglicht dies. Die $L_{1}$ schwebt gleich einem abfallenden Buchenblatt mit einer Sinkgeschwindigkeit von nur 0,54 m pro Sekunde zu Boden.

\section{EINLEITUNG}

Die Kleine Fichtenquirlschildlaus oder Kleine Lecanie (Physokermes hemicryphus DALM.) ist die sicherste und deswegen auch wichtigste Honigtauerzeugerin in Österreich und anderen Fichtentrachtgebieten Mitteleuropas (Ruttner 1960, Fossel 1960). Wirtsp flanze ist vorwiegend Picea excelsa (und andere Picea-Arten) und untergeordnet Abies alba. Trotz des häufigen Vorkommens dieser Honigtauerzeuger treten im Wechsel der Jahre und Gebiete in der Tracht große Schwankungen auf. Es wird daher nach einer PrognoseMöglichkeit dieser Honigtautracht gesucht. Um aber zu einer brauchbaren Prognose zu kommen, mußte u.a. auch die Art und Weise der Ausbreitung der Kleinen Lecanie untersucht werden.

Es wurde schon wiederholt darauf hingewiesen, daß zur Zeit des Schlüpfens und Ausschwärmens der $L_{1}$ das Wetter einen z. T. bedeutenden Einfluß auf deren Überleben (und damit auf die nächstjährige Tracht) ausübt (Sснмuтterer 1965, Gontarski 1940, Hölzl 1956, Fossel 1960).

Aus den zwei Brutkammern eines einzigen nicht parasitierten Muttertieres der Kleinen Lecanie schlüpfen nach Schmutteren (1956) um 200 (38 - 335) $\mathrm{L}_{1}$ - nach eigenen Zählungen 140 bis 520. Davon hat der Großteil nicht die Möglichkeit, in den benachbarten Quirlen Unterschlupf zu finden. Maximal wurden 20-25 angesaugte Larven der Kleinen Lecanie in einem Quirl gezählt, von denen aber während der Reifung zur ausgewachsenen Imago noch viele aus Platzmangel zugrunde gehen (Schmutterer 1965). Einem weiten Fußmarsch sind ob der Kleinheit und relativ geringen Beweglichkeit (Schmutterer 1965) zumindest innerhalb eines Baumes Grenzen gesetzt. Ein aktives Überwechseln von einem Baum auf einen anderen ist ausgeschlossen, da es keine geflügelte weibliche Form gibt (als einziges Entwicklungsstadium der Kleinen Lecanie ist die männliche Imago geflügelt). 
Die Ausbreitung der Junglarven $\left(\mathrm{L}_{1}\right)$ der Kleinen Lecanie unmittelbar nach dem Verlassen der mütterlichen Hülle geht innerhalb eines bereits besetzten Baumes auf dem Wege des Fußmarsches vor sich (Schmutterer 1956, Fossel 1960, KLoft 1966). Dies ist zur Schlüpfzeit leicht zu beobachten. Dagegen ist die Ausbreitung von Baum zu Baum eine zum größten Teil noch ungeklärte Frage.

Nach Kloft und Schmutterer (1957) geht die Ausbreitung verschiedener Cocciden durch den Wind vor sich; aber auch andere Insekten (z. B. Ameisen) werden als Ausbreitungshilfe angeführt. Auch nach Fosses (mündliche Mitteilung) haben die Ameisen einen positiven Einfluß auf die Populationsdichte der Kleinen Lecanie - aber sehr wahrscheinlich durch Schutz vor Parasiten. Nach Ruppertshofen (1967) sollen bei der Kleinen Lecanie auch verschiedene Spinnen einen entscheidenden Einfluß auf die Ausbreitung haben. SchmutreRER (1965) erwähnt, daß starker, trockener Wind nicht nur als Mortalitätsfaktor (Austrocknung und Verwehung) bei der Kleinen Fichtenquirlschildlaus auftritt, sondern auch der Verbreitung Vorschub leisten kann.

Der asselähnliche, flachgedrückte Habitus der Erstlarven von 0,6-0,65 mm Länge und 0,25-0,30 mm Breite (Schmutterer, 1956) scheint zum Schweben in der Luft ausgezeichnet geeignet zu sein (wie dies nachfolgend angeführte Ergebnisse eindeutig zeigen).

Es wurden verschiedene Faktoren, die einen Einfluß auf die Ausbreitung haben können, auf ihre Auswirkung untersucht. Ferner wurden, um die Rolle des Windes für die Ausbreitung zu klären, in den Jahren 1968, 1969 und 1970 Feldexperimente durchgeführt.

\section{METHODIK}

Die Beobachtungen über die Auswirkungen der Umweltein flüsse auf den Schlupfvorgang wurden im Freiland oder in Labor und Klimakammer gemacht.

Um die Sinkgeschwindigkeit einer $L_{1}$ der Kleinen Lecanie im freien Fall festzustellen, wurde folgendermaßen vorgegangen : In einem lichtundurchlässigen Terrarium, das mit einer lichtdurchlässigen Glasplatte abgedeckt war, wurden die $L_{t}$ bei normaler Zimmertemperatur aus eingesammelten Muttertieren zum Schlüpfen gebracht. Die frisch geschlüpften $L_{1}$ sind sehr stark positiv phototaktisch (ScHMUTrERER, 1956), sie streben dem Lichte zu und sammelten sich so an der Unterseite der zur Abdeckung verwendeten Glasplatte. Diese Glasplatte wurde in einem geschlossenen Raum auf 1 bzw. $2 \mathrm{~m}$ Höhe gebracht. Die $\mathrm{L}_{1}$, die an der Unterseite haften bleiben, wurden von der Glasplatte abgestoßen und ihr Herabsinken zur Erde im starken Gegenlicht vor einem schwarzen, lichtundurchlässigen Vorhang beobachtet und die Falldauer mit der Stoppuhr festgehalten.

Zur Feststellung der vom Wind vertragenen $L_{1}$ der Kleinen Lecanie wurden Fanggeräte in verschiedenen Entfernungen von lecanienbesetzten Fichtenbeständen ausgelegt. Hiezu wurden Glastafeln in der willkürlich gewählten Größe von $32 \times 19 \mathrm{~cm}$ mit einer dünnen Schicht Raupenleim überzogen. Eine Glastafel wurde dabei einfach mit einer Spachtel dünn mit Raupenleim bestrichen. Danach wurde ein Blatt Papier aufgelegt, mit der Hand fest angepreßt und wieder abgezogen. Dieses lückenlos mit Raupenleim behaftete Papier wurde auf eine zweite, noch trockene Glasplatte aufgelegt und wieder fest angepreßt. Auf die erste Glasplatte wurde neuerdings ein Blatt Papier gelegt. So waren zwei Platten fertig präpariert. Man erreicht so eine dünne, gleichmäßige Leimschicht, damit größere Insekten nicht haften bleiben. Das Papier ergab zugleich einen Schutz vor Verunreinigungen beim Transport. 
Diese so präparierten Glasplatten wurden dann am gewünschten Ort horizontal mit der Leimschicht nach oben ausgelegt, indem man einfach das Papier abzog.

Die Glasplatten wurden nur nach Beginn (Periode I) und gegen Ende der Schlupfzeit (Periode II) 1 - 2 Wochen (sishe Tab. 3) jeweils auf Platz A und auf Platz $B$ im Wald (0 m), 5 m von der Baumtraufe, $50 \mathrm{~m}$ und $500 \mathrm{~m}$ vom Wald entfernt ausgelegt. Um die Zahl der vom Winde verschleppten $L_{1}$ quantitativ feststellen zu können, müßte man während der ganzen Schlupfzeit Glasplatten auslegen, die jode Woche zu erneuern wären. Dies konnte aber arbeitsmäßig nicht bewältigt werden. An einem Ort wurden jeweils 3 Glasplatten ausgelegt, um die Wahrscheinlichkeit eines Fanges zu vergrößern.

Platz A (Abb. 1) liegt auf dem Hochplateau der Böhmischen Masse, ca. $20 \mathrm{~km}$ nördlich Ybbs/Donau. Es handelt sich um ein mehr freies Gelände mit sanften Erhebungen (550 bis $900 \mathrm{~m}$ über $\mathrm{NN}$ ), die fast ausschließlich mit Fichte bestockt sind.

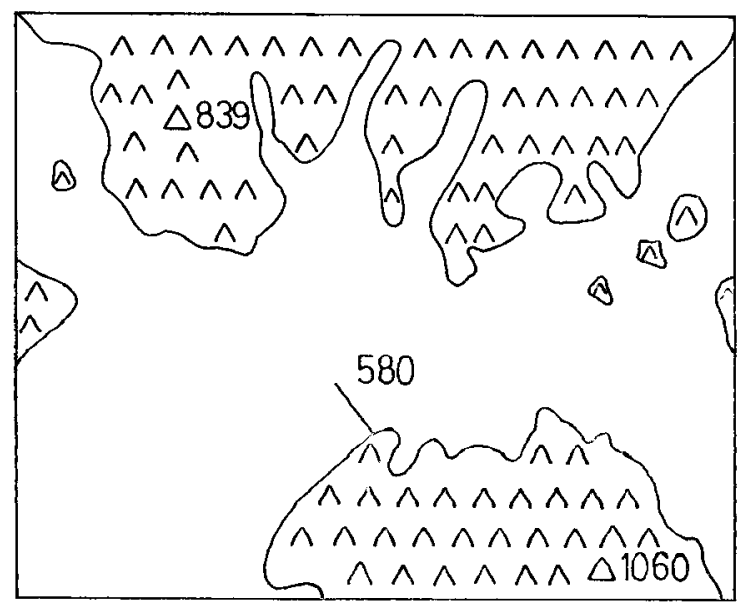

$1000500 \quad 0 \quad 1000 \mathrm{~m}$

Авв. 1. - Skizze der geographischen Lage von Platz A.

Auf der gerade ausgezogenen Strecke zwischen Wald und der Angabe der Meereshöhe von $580 \mathrm{~m}$ wurden die Glasplatten in verschiedenen Entfernungen vom Wald ausgelegt (siehe Text!).

Fig. 1. - Croquis montrant la situation géographique de l'emplacement $A$.

Les plaques de verre ont été placées à diverses distances le long de la droite partant de la forêt pour aboutir au point marqué $580 \mathrm{~m}$ au-dessus du niveau de la mer. (Explications dans le texte).

Platz B (Abb. 2) ist ein Talkessel (617 m über NN) in den Nördlichen Kalkalpen Niederösterreichs bei Lunz/See. Die steil ansteigenden Berge (bis $1882 \mathrm{~m}$ über NN) sind ebenfalls hauptsächlich mit Fichte bestockt. Platz A wies 1968 und 1969 einen sehr starken Lecanienbesatz auf. 1970 ging der Besatz hier stark zurück. Platz B hatte 1968 und 1969 relativ schwachen Besatz. 1970 war hier dagegen der Besatz außergewöhnlich stark.

Die Glasplatten wurden nach der Abnahme unter einem Präpariermikroskop mit ca. 20facher Vergrößerung nach $L_{1}$ untersucht und ausgezählt.

\section{ERGEBNISSE}

\section{1. - Schlüpfen der Junglarven}

Das Wetter ist für die Ausbreitung der Kleinen Lecanie während ihrer Schlupfzeit von großer Bedeutung. Es wurde übereinstimmend mit Schmutre- 


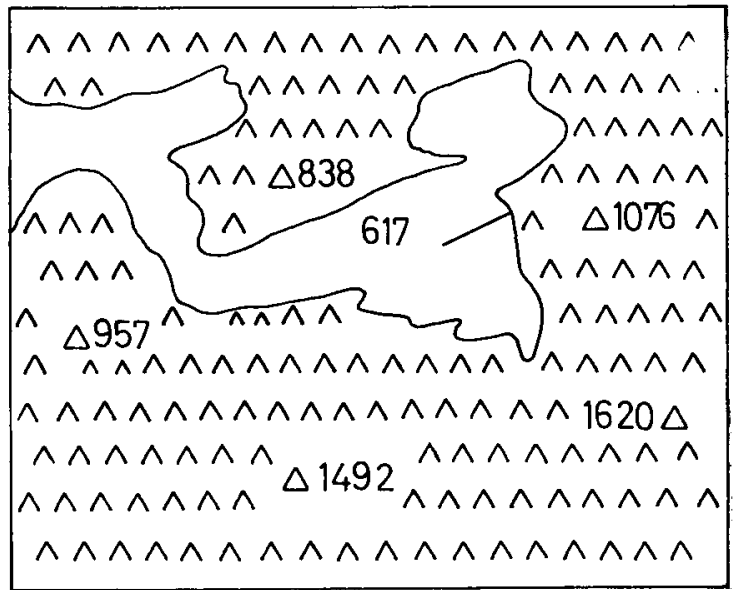

$1000500 \quad 0 \quad 1000 \mathrm{~m}$

Aвв. 2. - Skizze der geographischen Lage von Platz B.

Auf der gerade ausgezogenen Strecke zwischen Wald und der Angabe der Meereshöhe von $617 \mathrm{~m}$ wurden die Glasplatten in verschiedenen Entfernungen vom Wald ausgelegt (siehe Text!).

Fıg. 2. - Croquis montrant la situation géographique de l'emplacement $B$.

Les plaques de verre ont été placées à diverses distances le long de la droite partant de la forêt pour aboutir au point marqué $617 \mathrm{~m}$ au-dessus du niveau de la mer. (Explications dans le texte).

RER (1965) beobachtet, daß die $L_{1}$ nur bei wärmerem und trockenem Wetter schlüpfen. Schm utTerer nennt als Temperaturoptimum 24-28 oC. Nach seinen Beobachtungen stellt hohe rel. Luftfeuchtigkeit in Verbindung mit Niederschlägen einen der wichtigsten Mortalitätsfaktoren der $L_{1}$ dar. GonTarski (1940), HölzL (1956) machten ähnliche Beobachtungen. Fosses (mündl. Mitteilung) beobachtete an bei Zimmertemperatur in $W$ asser eingefrischten Fichtenzweigen mit schlupfreifen $L_{1}$ folgendes : Standen die Zweige in der Sonne, kam es zu einem fast überstürzten Verlassen der mütterlichen Hülle. Wurden die Zweige aber in den Schatten gestellt, so war kein Ausschlüpfen festzustellen.

Dies könnte mit dem von SchmuTTERer erwähnten stark positiv phototaktischen Verhalten zu erklären sein. Fossel beobachtete dabei, daß der Analspalt in der Sonne offen stand, im Schatten aber geschlossen war. Sie führte diese Erscheinung nur auf Temperatur - und Lichteinwirkung zurück. Bei genauerer Untersuchung dieses Verhaltens stellte sich aber heraus, daß Lichtintensität und Temperatur bei gleichbleibenden Feuchtigkeitsverhältnissen der toten mütterlichen Hülle keinerlei Einfluß auf die Spaltöffnung haben. Nur der wechselnde Feuchtigkeitszustand, unabhängig von Temperatur - und Lichteinwirkung, bewirkt ein Öffnen und Schließen des Analspaltes. Es 
handelt sich hier um eine rein hygroskopische Eigenschaft der toten mütterlichen Hülle. Es zeigte sich, daß in vollkommen nassem Zustand der Hülle der Analspalt fast vollkommen geschlossen ist (Abb. 4). Im trockenen Zustand bei einer rel. Luftfeuchtigkeit unter 80-85 \% ist der Spalt mehr oder weniger weit geöffnet (Abb. 3). Der Unterschied in der Spaltbreite zwischen nassem

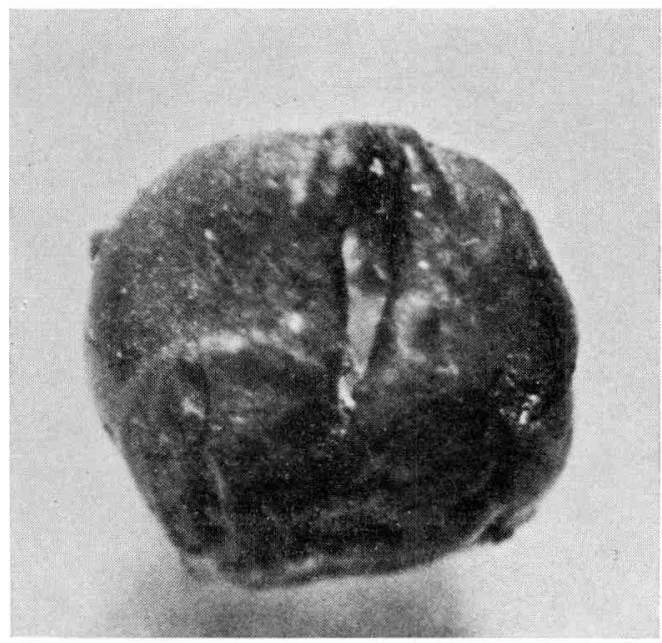

Aвв. 3. - Hülle einer Kleinen Lecanie im trockenen Zustand mit offenem Analspalt.

Frg. 3. - Coque d'un petit Lecanium à l'état sec avec le sillon anal ouvert.

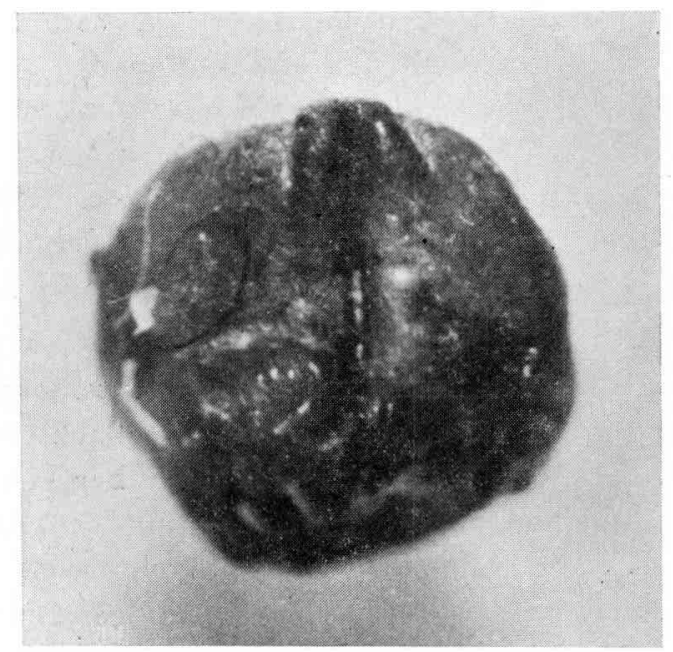

Aвв. 4. - Hülle einer Kleinen Lecanie im feuchten Zustand mit geschlossenem Analspalt.

Fic . 4. - Coque d'un pétit Lecanium à l'état humide avec le sillon anal fermé. 
und trockenem Zustand beträgt im Durchschnitt von 70 Tieren $0,16 \mathrm{~mm}$; die Dicke einer $L_{1}$ beträgt ca. $0,08-0,10 \mathrm{~mm}$. Der Analspalt erscheint aus der Sicht der Larven je nach den äußeren Feuchtigkeitsverhältnissen als offene oder geschlossene Tür.

Diese Messungen wurden nur im Labor gemacht, doch ist diese hygroskopische Eigenschaft unter natürlichen Bedingungen genauso zu beobachten. Ein z. B. 0,2 mm weit offener Spalt schließt sich in der Regel innerhalb von 2-5 Minuten (u. U. schon in 30 Sekunden), sobald auf die Hülle ein Tropfen Wasser fällt. Bei einer rel. Luftfeuchtigkeit über $80 \%$ (die Reaktion der einzelnen Hüllen ist verschieden) beginnt sich der Spalt zu schließen und ist bei Erreichung des Taupunktes meist geschlossen.

Das Öffnen eines geschlossenen Spaltes kann selbst bei $20^{\circ} \mathrm{C}$ und $4.0 \%$ rel. Luftfeuchtigkeit bis zu 30 Minuten dauern. Legt man eine nasse Hülle mit geschlossenem Spalt bei Zimmertemperatur auf ein trockenes Löschblatt, so geht die Öffnung des Spaltes bei einer rel. Luftfeuchtigkeit von $40 \%$ in 10 bis 20 Minuten vor sich. Bei einer rel. Luftfeuchtigkeit von $65-70 \%$ hingegen dauert es bis zu 3 Stunden, bis ein geschlossener Spalt ganz geöffnet ist. Unter natürlichen Verhältnissen müssen die Rinde des Zweiges und die Quirlschuppen mittrocknen, was die Öffnung des Spaltes sehr verzögern kann. Hier spielt auch die austrocknende Wirkung des Windes eine große Rolle. Auch Rußtau verzögert die Öffnung, bzw. kann sie ganz verhindern. Von den 70 untersuchten Hüllen war die Änderung der Spaltöffnung durch Pilzeinwirkung bei 7 kleiner als $0,1 \mathrm{~mm}$. Die $\mathbf{L}_{1}$ gehen dann in der mütterlichen Hülle zugrunde, was auch SChmutterer (1965) als Mortalitätsfaktor angibt.

Über die Witterungsverhältnisse (Temperatur, Luftfeuchte und Niederschlag), die Verbreitung und Fangergebnisse beeinflussen, wurden in den Fangperioden Aufzeichnungen gemacht. Als Schönwettertage (Tab. 3) wurden nur niederschlagsfreie Tage mit einem Temperaturmaximum von über $20^{\circ} \mathrm{C}$ und einem Mittagswert der rel. Luftfeuchtigkeit von unter $70 \%$ berücksichtigt. Kurze Gewitterregen wurden nicht einbezogen, sofern der übrige Tagesablauf Schönwetter aufwies. Die Aufzeichnung der Windverhältnisse war nur auf Stand B möglich.

Der freie Fall der $\mathrm{L}_{1}$ der Kleinen Lecanie gleicht dem Fall eines Buchenblattes im Herbst : Die $L_{1}$ schwebt hin - und herpendelnd zur Erde. Sie legt dabei im Durchschnitt $0,54 \mathrm{~m}$ pro Sekunde zurück. Es hat sich zwischen der Fallhöhe von $1 \mathrm{~m}$ und $2 \mathrm{~m}$ kein Unterschied in der Sinkgeschwindigkeit gezeigt.

Die Schlupfzeit zog sich auf Platz A und B 1968 und 1969 von Ende Juli (ab 25.7.) bis Mitte September hin. Sich frei bewegende Lecanienlarven wurden jeweils noch Ende Oktober beobachtet. 1970 war die Schlupfzeit 2 Wochen später. 


\section{2. - Verbreitung der $L_{1}$ im Gelände}

Die Fangergebnisse mit den präparierten Glasplatten waren besonders 1968 und 1969 in der Periode $I$ auf Platz A überraschend gut. 1970 waren die Fangergebnisse auf Platz $B$ in der Periode II am besten (Tab. 1 und 2).

Tabelle 1. - Fangergebnisse auf Platz $A$

Tableau 1. - Résultats des captures à l'emplacement $A$

\begin{tabular}{|c|c|c|c|c|c|c|c|}
\hline \multirow{3}{*}{$\begin{array}{l}\text { Entfernung } \\
\text { vom Wald } \\
\text { in m } \\
\text { Distance de } \\
\text { la forêt en m }\end{array}$} & \multicolumn{7}{|c|}{$\begin{array}{c}\text { Gefangene } \mathrm{L}_{1} \text { pro } \mathrm{m}^{2} \\
\mathrm{~L}_{1} \text { capturées par } \mathrm{m}^{2}\end{array}$} \\
\hline & \multicolumn{3}{|c|}{ Periode I } & \multicolumn{3}{|c|}{ Periode II } & \multirow{2}{*}{$\begin{array}{l}\text { Durch } \\
\text { schnitt } \\
\text { Moyenne }\end{array}$} \\
\hline & 1968 & 1969 & 1970 & 1968 & 1969 & 1970 & \\
\hline 0 & 2556 & 2298 & 256 & 56 & 24 & 66 & 876 \\
\hline 5 & 2278 & 1293 & 48 & 32 & 0 & 44 & 616 \\
\hline 50 & 333 & 462 & 24 & 0 & 0 & 48 & 145 \\
\hline 500 & 41 & 25 & 0 & 0 & 0 & 16 & 14 \\
\hline
\end{tabular}

Tabelle 2. - Fangergebnisse auf Platz B

TABleaU 2. - Résultats des captures à l'emplacement B

\begin{tabular}{|c|c|c|c|c|c|c|c|}
\hline \multirow{3}{*}{$\begin{array}{l}\text { Entfernung } \\
\text { vom Wald } \\
\text { in } \mathrm{m} \\
\text { Distance de } \\
\text { la forêt en m }\end{array}$} & \multicolumn{7}{|c|}{$\begin{array}{c}\text { Gefangene } \mathrm{L}_{1} \text { pro } \mathrm{m}^{2} \\
\mathrm{~L}_{1} \text { capturées par } \mathrm{m}^{2}\end{array}$} \\
\hline & \multicolumn{3}{|c|}{ Periode I } & \multicolumn{3}{|c|}{ Periode II } & \multirow{2}{*}{$\begin{array}{l}\text { Durch } \\
\text { schnitt }\end{array}$} \\
\hline & 1968 & 1969 & 1970 & 1968 & 1969 & 1970 & \\
\hline 0 & 132 & 441 & 48 & 0 & 110 & 203 & 156 \\
\hline 5 & 44 & 88 & 32 & 0 & 56 & 80 & 50 \\
\hline 50 & 0 & 81 & 24 & 0 & 52 & 16 & 29 \\
\hline 500 & 0 & 16 & 8 & 0 & 16 & 64 & 18 \\
\hline
\end{tabular}

Hin und wieder waren Glasplatten ungewollt auch an der Unterseite teilweise mit Raupenleim beschichtet worden. An solchen Stellen wurden besonders im Wald und $5 \mathrm{~m}$ vom Waldrand wiederholt gefangene $L_{1}$ festgestellt. Dies bedeutet, daß abgefallene $\mathrm{L}_{1}$ auch am Erdboden ihre $W$ anderung fortsetzen und so an den klebrigen Stellen unterseits haften bleiben. Oberseits ist kaum eine Zuwanderung zu Fuß anzunehmen, da an den Rändern der Glasplatten kein gehäufter Fang zu verzeichnen war. 
1969 und 1970 wurde noch jeweils im März an wärmeren Tagen (Temperaturmaximum um $+15^{\circ} \mathrm{C}$ ) mit starker Sonneneinstrahlung auf Platz $B$ eine rege Wandertätigkeit der Zweitlarven $\left(L_{2}\right)$ an den Fichtenzweigen beobachtet. Besonders leicht festzustellen war dies an 8 - bis 10 jährigen Jungfichten mit starkem Besatz an Großer Lecanie (Physokermes piceae DALM.). Die $L_{2}$ wanderten besonders an der sonnenbeschienenen Seite der Zweige auf und ab und waren offenbar auf der Suche nach einem neuem Saugort. Viele von diesen $\mathrm{L}_{2}$ fielen dabei zu Boden. Es färbte sich der Schnee (um diese Jahreszeit liegt im Beobachtungsgebiet noch regelmäßig Schnee) innerhalb einiger warmer Tage durch abgefallene $L_{2}$ leicht rot. Bei der Kleinen Lecanie konnte wohl auch eine rege Wanderung zur gleichen Zeit beobachtet werden, nur war der starke Abfall in den Schnee nicht zu verzeichnen. Im Vergleich zur Großen Lecanie konnten von der Kleinen Lecanie nur wenige $L_{2}$ im Schnee gefunden werden.

Diese Wandertätigkeit der $\mathrm{L}_{2}$ scheint hauptsächlich von der Temperatur abhängig zu sein. Bringt man nämlich im Winter mit $L_{2}$ besetzte Fichtenzweige in einen warmen Raum, so begibt sich immer ein kleiner Teil dieser $\mathbf{L}_{2}$ auf Wanderschaft. Dies trifft auch bei sehr schwachem Besatz zu. Klappt man z. B. unter einem Präpariermikroskop die Quirlschuppen zurück, so kann man gut beobachten, wie ein Teil der $L_{2}$ mühsam ihren Rüssel herauszieht und sich unter Umständen ein neues, geschützteres Quartier sucht. Die anderen $L_{2}$ verlassen ihren Saugort nicht.

\section{DISKUSSION}

Von den Witterungseinflüssen spielen bei der Verbreitung der Erstlarven Temperatur, Wind, rel. Luftfeuchtigkeit und Niederschlag eine bedeutende Rolle. Der Feuchtigkeitszustand der mütterlichen Hülle ist für die Schlüpfmöglichkeit und den Schlüpftermin der Kleinen Lecanie von Bedeutung.

Die Temperatur hat außer in Zusammenhang mit der rel. Luftfeuchtigkeit und mit der Feuchtigkeit der Hülle lediglich einen Einfluß auf die Beweglichkeit der $\mathrm{L}_{1}$. Der Wind ermöglicht besonders an warmen und trockenen Tagen die Ausbreitung von Baum zu Baum, überdies beschleunigt er durch seine austrocknende Wirkung sehr stark die Öffnung des Analspaltes.

Der Feuchtigkeitszustand der mütterlichen Hülle, bedingt durch Niederschlag, Tau oder rel. Luftfeuchtigkeit, kann wegen der hygroskopischen Eigenschaft der Hülle einerseits Schutz vor Abwaschung oder zu starker Befeuchtung sein, andererseits aber bei zu langer Sperre zum Tod der $L_{1}$ führen. Bei Regen und hoher rel. Luftfeuchtigkeit herrscht sozusagen Ausgan zssperre. Das heißt, die Erstlarven sind automatisch gezwungen, sich nicht zur Unzeit auf Wanderschaft zu begeben. Dies kann ihnen nur bei langandauerndem Schlechtwetter zum Verhängnis werden. 
Die Vitalität der $L_{1}$ geht nämlich bei Verhinderung des Schlupfes nach Schm UTterer (1965) sehr rasch zurück. Man findet oft Hüllen alter Tiere, die voll toter $L_{1}$ sind. Die Ansicht erfahrener Imker, daß Waldstriche, die häufig im Nebelbereich liegen, schlechtere Fichtentrachtgebiete sind, wird nach diesen Erfahrungen erhärtet.

Die Versuche über die Ausbreitung von Baum zu Baum ergaben, daß eine Verschleppung der $L_{1}$ der Kleinen Lecanie durch den Wind in sehr starkem Ausmaß gegeben ist. Ein Vergleich zwischen den Fangzahlen in den verschiedenen Abständen vom Wald zeigt allerdings, daß die Hauptmasse der $L_{1}$ fast direkt vom Baum fällt. Unter einem besonders stark besetzten Fichtenbestand wurden 1968 in der Periode I (1.8. - 8.8.) auf Platz A sogar $4280 \mathrm{~L}_{1}$ pro $\mathrm{m}^{2}$ gefangen. Das ergibt knapp über 200000 abgefallene $\mathrm{L}_{1}$ innerhalb einer Woche unter der Krone einer alten Fichte (ca. 8 m Durchmesser)!

Jedoch waren auch die Fangergebnisse in den verschiedenen Abständen vom Wald überraschend hoch. So wurden noch in der Entfernung von $500 \mathrm{~m}$ vom Wald an beiden Plätzen in den zwei relativ kurzen Perioden durch drei Jahre hindurch durchschnittlich $16 \mathrm{~L}_{1}$ pro $\mathrm{m}^{2}$ gefangen. Das größte Fangergebnis in $500 \mathrm{~m}$ Entfernung vom Wald wurde 1970 auf Platz B in der Periode II (8.9. - 16.9.) mit $64 \mathrm{~L}_{1}$ pro $\mathrm{m}_{2}$ erzielt. Wenn man hiermit die Oberfläche einer freistehenden, 15 - 20 jährigen, kegelförmigen Jungfichte (ca. $16 \mathrm{~m}^{2}$ ) vergleicht, so kann man den Schluß ziehen, daß ein Neubesatz schon innerhalb einer Woche mit Sicherheit erfolgt. Andererseits zeigt sich aber, daß bei dieser Verschleppung durch den Wind die Verlustquote ungemein groß ist.

Wenn man die Durchschnittswerte der Fangergebnisse aus beiden Perioden von Platz A und Platz $B$ jeweils im Wald und $500 \mathrm{~m}$ vom Wald vergleicht, so zeigt sich auf Platz B eine auffallende Verflachung (vergleiche Tab. 1 und 2). Dies wird vor allem auf die geographische Lage zurückzuführen sein. Platz B ist unmittelbar von steilen, bewaldeten Bergen mit einer rel. Höhe bis zu $1000 \mathrm{~m}$ umgeben, während, wie schon erwähnt, Platz A wesentlich freier liegt. Auf Platz B hat um 14 Uhr häufig eine Windgeschwindigkeit von 3 (nach der Beaufort-Skala) geherrscht (Tab. 4). Nimmt man bei einem älteren Fichtenbestand eine mittlere Fallhöhe der Erstlarven $\left(L_{1}\right)$ von $12,5 \mathrm{~m}$ an, so ergibt sich im ebenen Gelände bei einer Windgeschwindigkeit von 3 (durchschnittlich $4,3 \mathrm{~m}$ pro Sekunde) schon eine Abtrift von ca. $100 \mathrm{~m}$, bis die $\mathrm{L}_{1}$ am Boden landet. Bei einer Windstärke von 4 nach der Beaufort-Skala (durchschnittlich 6,3 m pro Sekunde) ergibt sich schon eine Abtrift von $146 \mathrm{~m}$ im ebenen Gelände. Gerade im Gebirge ergeben sich durch die Hanglage meist viel größere Fallhöhen (Platz B), außerdem entstehen durch Erwärmung und Föhn häufig starke Aufwinde. Dadurch kann die Abtrift stark vergrößert werden.

Nach den bisherigen Erfahrungen hat die Wetterlage beim Massenwechsel 
TABELLE 3 .- Anzahl der Schönwettertage (Temperatur-Max, über 200 C und rel. Luftfeuchtigkeit um 14 Uhr unter $70 \%$ ) in den einzelnen Perioden und Jahren

TABLEAU 3. - Nombre de jours de beau temps (température max. au dessus de $20^{\circ}$ Cet humidité relative à 14 h inférieure à $70 \%$ ) dans les différentes périodes et années

\begin{tabular}{|c|c|c|c|c|c|c|}
\hline \multirow{3}{*}{$\begin{array}{l}\text { Jahr } \\
\text { Année }\end{array}$} & \multicolumn{3}{|c|}{ Periode I } & \multicolumn{3}{|c|}{ Periode II } \\
\hline & \multirow{2}{*}{$\begin{array}{c}\text { Dauer } \\
\text { von-bis } \\
\text { Durée } \\
\text { du - au }\end{array}$} & \multicolumn{2}{|c|}{$\begin{array}{l}\text { Schönwettertage } \\
\text { Jours de beau temps }\end{array}$} & \multirow{2}{*}{$\begin{array}{c}\text { Dauer } \\
\text { von-bis } \\
\text { Durée } \\
\text { du - au }\end{array}$} & \multicolumn{2}{|c|}{$\begin{array}{l}\text { Schönwettertage } \\
\text { Jours de beau temps }\end{array}$} \\
\hline & & $\begin{array}{c}\text { Platz A } \\
\text { Emplacement A }\end{array}$ & $\begin{array}{c}\text { Platz B } \\
\text { Emplacement B }\end{array}$ & & $\begin{array}{c}\text { Platz A } \\
\text { Emplacement A }\end{array}$ & $\begin{array}{c}\text { Platz B } \\
\text { Emplacement B }\end{array}$ \\
\hline 1968 & $1-8-8-8$ & 8 & 6 & $29-8-5-9$ & 6 & 5 \\
\hline 1969 & $28-7-8-8$ & 9 & 9 & $3-9-11.9$ & 6 & 6 \\
\hline 1970 & $9-8-25-8$ & 9 & 7 & $8-9-16-9$ & 6 & 6 \\
\hline
\end{tabular}

TABELLE 4. - Windstärke nach der Beaufort-Skala an den Schönwettertagen in den einzelnen Jahren und Perioden auf Platz $B$

Tableau 4. - Force du vent dans l'échelle de Beaufort au cours des journées de beau temps dans les différentes périodes et aussi à l'emplacement $B$

\begin{tabular}{|c|c|c|c|c|c|c|}
\hline \multirow{2}{*}{$\begin{array}{l}\text { Jahre } \\
\text { Année }\end{array}$} & \multicolumn{3}{|c|}{$\begin{array}{c}\text { Periode I } \\
\text { Windstärke von } \\
\text { Force du vent }\end{array}$} & \multicolumn{3}{|c|}{$\begin{array}{c}\text { Periode II } \\
\text { Windstärke von } \\
\text { Force du vent }\end{array}$} \\
\hline & $0-2$ & $2-4$ & $\begin{array}{c}\text { über } 4 \\
\text { au-dessus de } 4\end{array}$ & $0-2$ & $2-4$ & $\begin{array}{c}\text { über } 4 \\
\text { au-dessus de } 4\end{array}$ \\
\hline 1968 & 2 & 4 & 0 & 2 & 3 & 0 \\
\hline 1969 & 2 & 7 & 0 & 1 & 3 & 2 \\
\hline 1970 & 2 & 3 & 2 & 2 & 2 & 2 \\
\hline
\end{tabular}

der Kleinen Lecanie einen bedeutenden Einfluß, obwohl die wichtigste Ausbreitung nach wie vor innerhalb eines bereits besetzten Baumes von der Hülle des Muttertieres aus zu Fuß zu den benachbarten Quirlen der Fichte erfolgen wird. Das zeitweise Auftreten von Massenvermehrungen der Kleinen Lecanie über ganz Mitteleuropa (mit Massenproduktion von Honigtau) kann dadurch erklärt werden, daß eine von Parasiten dezimierte, sich wieder erholende Population mit Hilfe des Windes sehr rasch eine gleichmäßige Verteilung über den ganzen Waldbestand erreicht.

Einen noch unbekannten, wahrscheinlich aber unbedeutenden Faktor stellt die Möglichkeit dar, daß die $\mathrm{L}_{2}$ im Frühjahr noch ihren Saugort wechseln kann. 1969 und 1970 dürfte diese Frühjahrswanderung durch die rel. Überbevölkerung verursacht worden sein. Es ist fraglich, ob dieser Wandertrieb im Frühjahr vor der letzten Häutung eine regelmäßige Erscheinung ist. Bisher wurde in der Literatur noch kein Hinweis gefunden. Weitere Beobachtungen in Jahren mit geringem Besatz sind erforderlich. 
Die hier gemachten Erfahrungen gelten vermutlich ebenso für die Große Lecanie oder Große Fichtenquirlschildlaus (Physokermes piceae DALM.), nur ist deren bienenwirtschaftliche Bedeutung sehr gering.

Eingegangen im März 1971.

Reçu pour publication en mars 1971 .

\section{DANK}

Für die Anregung und die wertvollen Hinweise zu dieser Arbeit möchte ich Frau Dr. A. Fossec herslichst danken. Auch der Biologischen Station Lunz am See sei für die Überlassung der meteorologischen Daten gedankt.

\section{RÉSUMÉ}

Les présentes recherches ont pour objet d'expliquer la propagation des larves du premier âge $\left(\mathrm{L}_{1}\right)$ de Physokermes hemicryphus d'un arbre à l'autre. L'in fluence de différents facteurs du milieu sur la propagation a été également étudiée. Le rôle du vent dans la propagation passive a été tout spécialement observé du fait qu'il n'existe pas de possibilités de propagation active des $L_{1}$ d'un arbre à l'autre (la forme ailée femelle manque).

A l'extérieur, au laboratoire et en chambre climatisée l'in fluence de la température, de la lumière et de l'humidité (précipitations et humidité relative) sur l'ouverture anale a été tout d'abord étudiée. Par ailleurs, la vitesse de chute de $L_{1}$ fraîchement écloses au laboratoire a été déterminée.

A l'extérieur on a essayé en ueux endroits de capturer les larves du premier âge entraînées par le vent. L'endroit A est un terrain plus ou moins accidenté, l'endroit B est une vallée étroite entourée de hautes montagnes. Les essais ont été faits en 1968, 1969 et 1970 après le début (période I) et vers la fin (période II) des éclosions des $L_{1}$. Les captures ont été obtenues au moyen de plaques de verre enduites d'une couche de glue aussi mince que possible. Les plaques de verre ainsi préparées furent déposées aux endroits $A$ et $B$ dans la forêt $(0 \mathrm{~m})$, à $5 \mathrm{~m}$ de la lisière des arbres, à $50 \mathrm{~m}$ et $500 \mathrm{~m}$ de la forêt. Après leur enlèvement les plaques de verre furent examinées au microscope et les $L_{1}$ furent dénombrées.

En accord avec Schmutterer (1956) on a observé que les $L_{1}$ fraîchement écloses présentent une forte phototaxie positive; leur sortie dépend de la température, l'optimum étant compris entre 24 et $28^{\circ} \mathrm{C}$. Les présentes recherches ont montré que l'ouverture anale de l'enveloppe maternelle morte se ferme lorsque l'humidité relative est élevée (au-dessus de 80-85\%) ou lorsqu'il pleut. A l'état sec elle est ouverte indépendamment de la température et de l'intensité lumineuse. La différence d'ouverture entre l'état mouillé et l'état sec atteint en moyenne, pour 70 animaux, $0,16 \mathrm{~mm}$ (En comparaison, l'épaisseur d'une $L_{1}$ est de 0,08 à $0,1 \mathrm{~mm}$ ). Lorsqu'une goutte d'eau tombe sur une enveloppe sèche et ouverte, celle-ci se referme très rapidement (2 à 5 minutes). L'ouverture est sensiblement plus lente (de 10 minutes à quelques neures). Ces propriétés hygroscopiques de l'enveloppe maternelle signifient pour les $\mathrm{L}_{1}$ une protection par temps humide contre une éclosion prématurée mais également, si la fermeture dure trop longtemps, une forte mortalité car leur vitalité décroît rapidement après la sortie de l'œuf.

La $L_{1}$ ne peut se propager activement en marchant qu'à la surface de l'arbre déjà occupé. Une propagation passive sur de grandes distances est réalisée par le vent, ce que permet la faible vitesse de chute qui est en moyenne de $0,54 \mathrm{~m}$ par seconde. $L_{a} L_{1}$ flotte et tombe au sol selon un mouvement pendulaire. 
Les tableaux 1 et 2 montrent les résultats des captures effectuées sur les plaques de verre enduites de glue à différentes distances de la forêt. Ces résultats montrent qu'en l'espace d'une semaine, même à une distance de $500 \mathrm{~m}$ d'une forêt peuplée de Lecanium la propagation sur un épicéa non encore peuplé est possible.

Chez les larves du second âge $\left(L_{2}\right)$ on a pu encore observer avant la dernière mue, en 1969 et 1970, par des journées chaudes de mars, une intense activité motrice. On ne sait pas encore si cette activité est uniquement en rapport avec la surpopulation.

La propagation la plus importante à la surface d'un arbre déjà peuplé a lieu de toute façon ( à pied ». Cependant, les pullulations de Lecanium, souvent constatées soudainement dans toute l'Europe centrale (avec production massive de miellat) peuvent s'expliquer par le fait qu'une population décimée par les parasites et qui se rétablit est capable, avec l'aide du vent, de retrouver rapidement une répartition régulière sur l'ensemble de la forêt.

\section{LITERATUR}

Fossec A., 1960. Die Fichtentracht. Bienenvater, 81, (7/8), 11-36.

Gontarski H., 1940. Beitrag zur Honigtaufrage. $Z$ ang. Ent., 27, 321-332.

Hölzx F., 1956. Warum Fehljahre bei Fichte und Tanne. Bienenvater, 77, (9), 304-305.

KLoFT W., 1965. Honigtauerzeuger des Waldes. Das Waldhonigbuch. Ehrenwirth, München.

Kloft W. u. Schmutterer H., 1957. Coccoidea. Handbuch der Pflanzenkrankheiten. V. 5 Aufl., 4. Lieferg., Parey, Berlin.

Ruppertshofen H., 1967. Biene und Waldhygiene. Imkerfreund, 22, (1), 2-5.

Ruttner F., 1960. Waldtracht und Waldtrachtbeobachtung in Österreich. Bienenvater, 81, $(7 / 8), 3-10$.

Schmutterer H., 1954. Ergebnisse neuerer Untersuchungen über die Fichtenquirlschildlaus Physokermes piceae (Schrk.). Verh. Dtsch. Ges. ang. Ent., 12. Mitgliedervers., Frankfurt 1952, 168-174.

Schmutteren H., 1956. Zur Morphologie, Systematik und Bionomie der Physokermes-Arten an Fichte (Homopt. Cocc.). Z.ang.Ent., 39, 445-466.

Schmutterer H., 1965. Zur Ökologie und wirtschaftlichen Bedeutung der PhysokermesArten (Homopt. Coccoidea) an Fichte in Süddeutschland. Z.ang.Ent., 56, 300-325.

Anschrift des Verfassers : H. Pechhacker, Bundeslehr- und Versuchsanstalt für Bienenkunde, Aussenstelle A-3293 Lunz/See, Niederösterreich. 